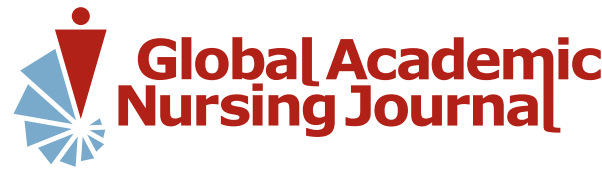

\section{A formação profissional do enfermeiro: entrelace entre ensino da virologia e SARS-CoV-2 em uma universidade pública}

\author{
The professional training of nurses: the link between teaching virology and SARS-CoV-2 in a public university \\ La formación profesional de enfermeras: el vínculo entre la enseñanza de virología y el SARS-CoV-2 en una \\ universidad pública
}

\author{
Miriam Marinho Chrizostimo ${ }^{1}$ \\ ORCID: 0000-0001-7498-4637 \\ Célia Pereira Caldas ${ }^{2}$ \\ ORCID: 0000-0002-5668-4398 \\ Nathalia Rodrigues de Brito ${ }^{1}$ \\ ORCID: 0000-0003-4125-3971 \\ Maritza Consuelo Ortiz Sánchez ${ }^{1}$ \\ ORCID: 0000-0002-6123-9846 \\ Deise Ferreira de Souza ${ }^{1}$ \\ ORCID: 0000-0002-4294-9957 \\ Maria Lelita Xavier ${ }^{2}$ \\ ORCID: 0000-0003-3014-733X \\ Amanda Ramiro Gomes da Silva ${ }^{1}$ \\ ORCID: 0000-0002-6383-5449 \\ Juliana de Oliveira Nunes da \\ Silva ${ }^{1}$ \\ ORCID: 0000-0002-9111-3806 \\ Victor Hugo Gomes Ferraz ${ }^{1}$ \\ ORCID: 0000-0003-4784-449X \\ ${ }^{1}$ Universidade Federal Fluminense. \\ Rio de Janeiro, Brasil. \\ 2Universidade do Estado do Rio de \\ Janeiro. Rio de Janeiro, Brasil.
}

\section{Como citar este artigo:}

Chrizostimo MM, Caldas CP, Brito NR, Sánchez MCO, Souza DF, Xavier ML, Silva ARG, Silva JON, Ferraz VHG. A formação profissional do enfermeiro: entrelace entre ensino da virologia e SARS-CoV-2 em uma universidade pública. Glob Acad Nurs. 2021;2(Spe.2):e119. https://dx.doi.org/10.5935/26755602.20200119

Autor correspondente: Miriam Marinho Chrizostimo E-mail: miriammarinho@hotmail.com

Editor Chefe: Caroliny dos Santos Guimarães da Fonseca Editor Executivo: Kátia dos Santos Armada de Oliveira

Submissão: 25-06-2021

Aprovação: 07-07-2021

\section{Resumo}

Objetivou-se analisar a formação do enfermeiro relacionada ao ensino da Virologia para a atuação em tempos de pandemia. Foi feita pesquisa descritiva, exploratória e qualitativa por meio de análise de conteúdo realizada nos meses de junho a agosto de 2020, servindo de subsídio para a construção de uma revisão integrativa pautada em leitura analítica, que contou com a apreciação de nove artigos, atendendo ao rigor metodológico. A orientação teórica teve o enfoque no desenvolvimento das competências de Philippe Perrenoud; nas Diretrizes Curriculares Nacionais para os cursos de graduação da área da saúde, que tratam sobre a formação profissional do enfermeiro; e no surgimento da pandemia causada pela SARS-COV2. Os dados mostraram que a formação do enfermeiro voltada para a atuação em tempos de pandemia emerge como categoria temática, onde o processo formativo desse profissional com relação ao ensino da Virologia apresenta-se como relevante. A construção da competência do enfermeiro é importante, pois ele atua junto ao paciente, que ocupa o papel de protagonista do atendimento, principalmente, em tempos de pandemia. Isso os torna capacitados para atender no SUS.

Descritores: Formação Profissional; Ensino; Enfermeiro; Virologia; Pandemias.

\section{Abstract}

The aim was to analyze the training of nurses related to the teaching of Virology to work in times of pandemic. Descriptive, exploratory, and qualitative research was carried out through content analysis carried out from June to August 2020, serving as a subsidy for the construction of an integrative review based on analytical reading, which included the appreciation of nine articles, considering the methodological rigor. The theoretical orientation focused on the development of competences of Philippe Perrenoud; in the National Curriculum Guidelines for undergraduate courses in the health area, which deal with the professional training of nurses; and in the onset of the pandemic caused by SARS-COV-2. The data showed that the training of nurses focused on acting in times of pandemic emerges as a thematic category, where the training process of this professional in relation to the teaching of Virology is presented as relevant. The construction of nurses' competence is important, as they work with the patient, who plays the leading role in care, especially in times of pandemic. This makes them able to serve in the SUS.

Descriptors: Professional Training; Teaching; Nurse; Virology; Pandemic.

\section{Resumén}

El objetivo fue analizar la formación de enfermeros relacionados con la enseñanza de Virología para trabajar en tiempos de pandemia. Se realizó una investigación descriptiva, exploratoria y cualitativa a través del análisis de contenido realizado de junio a agosto de 2020, sirviendo de subsidio para la construcción de una revisión integradora basada en la lectura analítica, que incluyó la valoración de nueve artículos, teniendo en cuenta el rigor metodológico. La orientación teórica se centró en el desarrollo de competencias de Philippe Perrenoud; en los Lineamientos Curriculares Nacionales para los cursos de pregrado en el área de la salud, que tratan de la formación profesional de enfermeras; y en el inicio de la pandemia causada por el SARSCOV-2. Los datos mostraron que la formación de enfermeros enfocados a actuar en tiempos de pandemia surge como una categoría temática, donde se presenta como relevante el proceso de formación de este profesional en relación a la docencia de Virología. La construcción de la competencia del enfermero es importante, ya que trabaja con el paciente, quien juega un papel protagónico en la atención, especialmente en tiempos de pandemia. Esto los capacita para asistir al SUS.

Descriptores: Formación Profesional; Enseñando; Enfermero; Virología; Pandemia. 


\section{Introdução}

Este estudo aborda a formação do enfermeiro, considerando o contexto da Pandemia. Seu propósito é problematizar o ensino da Virologia em uma universidade federal e dar relevância a esta disciplina. Uma vez que, o surgimento da SARS-COV-2 evidenciou o descompasso existente entre a formação dos profissionais de saúde e as necessidades de atenção que a população passou a demandar durante esse período.

Historicamente, a formação profissional do enfermeiro se vê envolta com a inclusão do Estado na conjuntura dos setores da saúde e da educação. Percebe-se que o impacto desta inserção influencia a sociedade, impulsiona o processo de desenvolvimento da Enfermagem e o marco conceitual da profissão, no que se refere à procura do processo saúde-doença da população; das características das doenças e de seus agravos; a evolução da ciência e da tecnologia ${ }^{1}$.

Durante o século XX no Brasil, os serviços de saúde pública na rede básica e, posteriormente, na rede hospitalar, evidenciaram a necessidade de formação de pessoal de Enfermagem com visão ampla e plena participação no controle de endemias, por meio do controle epidemiológico. Por outro lado, o ensino da Enfermagem foi marcado por reformas na educação superior que geraram diversificações nos currículos dos cursos de graduação ${ }^{1}$.

No século XXI, destaca-se o documento implementado em 2001 pela Câmara de Educação Superior do Conselho Nacional de Educação (CNE), que criou as Diretrizes Curriculares Nacionais (DCNs) para os cursos de graduação na área da saúde, incluindo: Enfermagem, Medicina e Nutrição. Nas DCNs, foram especificados novos critérios norteadores, o conteúdo geral e específico de cada área, assim como as competências e habilidades fundamentais para a formação do aluno. Desde então, existem diferentes atos normativos que guiam e regulam a formação profissional do enfermeiro, com vistas a coesão entre a formação profissional e o modelo de saúde, centrada na visão holística do paciente ${ }^{2}$.

Desse modo, o enfermeiro deve obter embasamento teórico e prático ao longo da sua formação, respaldada pelo diálogo, para construção de um profissional generalista, humanista, crítico e reflexivo, com foco nas diversas demandas da sociedade e do Sistema Único de Saúde (SUS), que abrange ações mais simples na atenção básica até à assistência de maior complexidade, como um transplante. Ressalta-se ainda, a importância do papel do enfermeiro, como integrante da equipe de saúde, no controle e assistência em situações de endemias / pandemias; assim como no campo da educação, pois ele possui papel intrínseco de educador seja de sua equipe ou da sociedade ${ }^{2}$.

Portanto, pesquisadores ${ }^{3}$ afirmam que a graduação em enfermagem objetiva inserir o estudante competência técnico-científica para o desenvolvimento de atividades assistenciais, gerenciais, de ensino e de pesquisa. Assim, é fundamental que durante a graduação, sejam estabelecidos princípios que irão nortear o processo de formação do enfermeiro e as disciplinas teóricas que devem ter conexão com as ações do cuidar de si e do outro. Seja de caráter preventivo, de recuperação e/ou de reabilitação, assim como de segurança dos trabalhadores da equipe de saúde. Os alunos devem agir como protagonistas do seu processo de aprender a ser enfermeiro.

Destaca-se como crucial, que no processo de formação do futuro enfermeiro sejam oferecidas na grade curricular, em caráter obrigatório, disciplinas da área de Microbiologia e, em especial a Virologia, foco do estudo devido a sua relevância particular que foi exacerbada no cenário atual de Pandemia. Conceitos básicos, riscos e ameaças, medidas de prevenção, funcionamento do sistema imunológico em uma infecção viral e mecanismos de disseminação precisam ser compreendidos e ensinados de maneira adequada. Visto que no momento, há um esforço mundial para evitar a propagação do vírus, seja de investimentos em pesquisas relacionadas à produção de medicamentos para curar os acometidos ou na produção de vacinas para prevenir a doença ${ }^{4}$.

De tal forma, este estudo destaca a temática do ensino de Virologia na formação profissional do enfermeiro. Sua pertinência consiste em mostrar a importância de formar profissionais capacitados para atender no SUS e preparar futuros educadores em saúde, preocupados em levar o conhecimento de maneira simples e eficaz. Outra motivação advém do momento que envolve também docentes e estudantes do curso de graduação de Enfermagem e da saúde de forma geral.

A síndrome respiratória aguda grave de Coronavírus 2 (SARS-COV-2) foi identificada pela primeira vez em dezembro de 2019, em Wuhan, na China. Esse é um novo tipo de Coronavírus que foi identificado como agente etiológico da doença Coronavírus 2019 (COVID-19). Sua disseminação foi caracterizada como Pandemia pela Organização Mundial de Saúde (OMS), em março de $2020^{5}$.

Desde então, diferentes tipos de medidas preventivas foram inseridos no cotidiano da população mundial para que a proliferação do Coronavírus seja contida. O objetivo é que os danos sejam mínimos, a transição para a normalidade seja rápida, enquanto aguarda-se o resultado de uma vacina. Nesse sentido, o estudo centra-se na formação e qualificação profissional, identificando a relevância do ensino do componente curricular Virologia em um curso de graduação de Enfermagem de uma universidade pública. De tal forma, seu objetivo é analisar a formação do enfermeiro relacionada à Virologia para atuar em tempos de pandemia.

\section{Metodologia}

Trata-se de um estudo descritivo e exploratório, com abordagem qualitativa, tendo como aporte teórico a teoria de Philippe Perrenoud, que enfoca o desenvolvimento das competências. O levantamento de dados foi realizado no período de junho a agosto de 2020. Inicialmente, foi feita a revisão integrativa da literatura, que serviu como subsídio para a análise e discussão do currículo do curso de graduação em Enfermagem, ministrado na Escola de Enfermagem Aurora de Afonso Costa (EEAAC), da Universidade Federal Fluminense (UFF). 
A formação profissional do enfermeiro: entrelace entre ensino da virologia e SARS-CoV-2 em uma universidade pública Chrizostimo MM, Caldas CP, Brito NR, Sánchez MCO, Souza DF, Xavier ML, Silva ARG, Silva JON, Ferraz VHG

A definição do tema e as informações extraídas em relação às evidências das produções científicas foram pautadas na pergunta norteadora sobre a formação e qualificação profissional ${ }^{6}$, que foi: Qual é a relevância do ensino do componente curricular Virologia nesse curso de graduação?

Assim, para o desenvolvimento da etapa de revisão integrativa do estudo, foram percorridas oito etapas: 1) identificação do tema e da formulação da questão norteadora; 2) estabelecimento dos critérios para inclusão e exclusão dos estudos; 3) definição das bases de dados da pesquisa; 4) definição dos recursos da busca bibliográfica; 5) definição das informações a serem extraídas dos estudos selecionados; 6) avaliação dos estudos incluídos; 7) interpretação dos resultados; e 8) apresentação a revisão ${ }^{7}$.

Quanto aos critérios estabelecidos para inclusão, foram: artigos científicos publicados nos idiomas português e inglês, com o recorte temporal no período dos 2015 e 2020, textos completos disponíveis on-line, de autores de diferentes áreas. Já os critérios de exclusão foram: o estudo bibliográfico de diversos tipos e documentos recuperados de maneira repetida nas diferentes bases de dados.
O Portal da Biblioteca Virtual em Saúde (BVS) foi a plataforma escolhida para a busca das produções científicas. Desse modo, recuperou-se artigos indexados em diferentes bases de dados, como: Literatura Latino-Americana e do Caribe em Ciências da Saúde (LILACS), Medical Literature Analysisand Retrievel System Online (MEDLINE) e Base de Dados de Enfermagem (BDENF - Enfermagem).

Foram utilizados os seguintes Descritores em Ciências da Saúde (DECs): Capacitação profissional; Estudantes de Enfermagem; Ensino; Virologia; e Microbiologia. Bem como, os descritores do Medical Subject Headings (MeSH): Professional training; Students, nursing; Virology, e Microbiology. Sendo empregados operadores booleanos "AND" e "OR" nas estratégias de busca, que permitiram a identificação inicial de 267.195 artigos (Figura 1).

No filtro de busca, foram aplicados os seguintes itens: coleção nacional e internacional; temas sobre gestão, educação e participação em saúde; assuntos principais de educação em enfermagem, educação baseada em competências, currículo e educação superior; publicações oriundas de países como o Brasil e dos continentes Europa e América do Norte, o que afunilou o achado para 145 artigos.

Figura 1. Resultado da estratégia de busca. Rio de Janeiro, RJ, Brasil, 2020

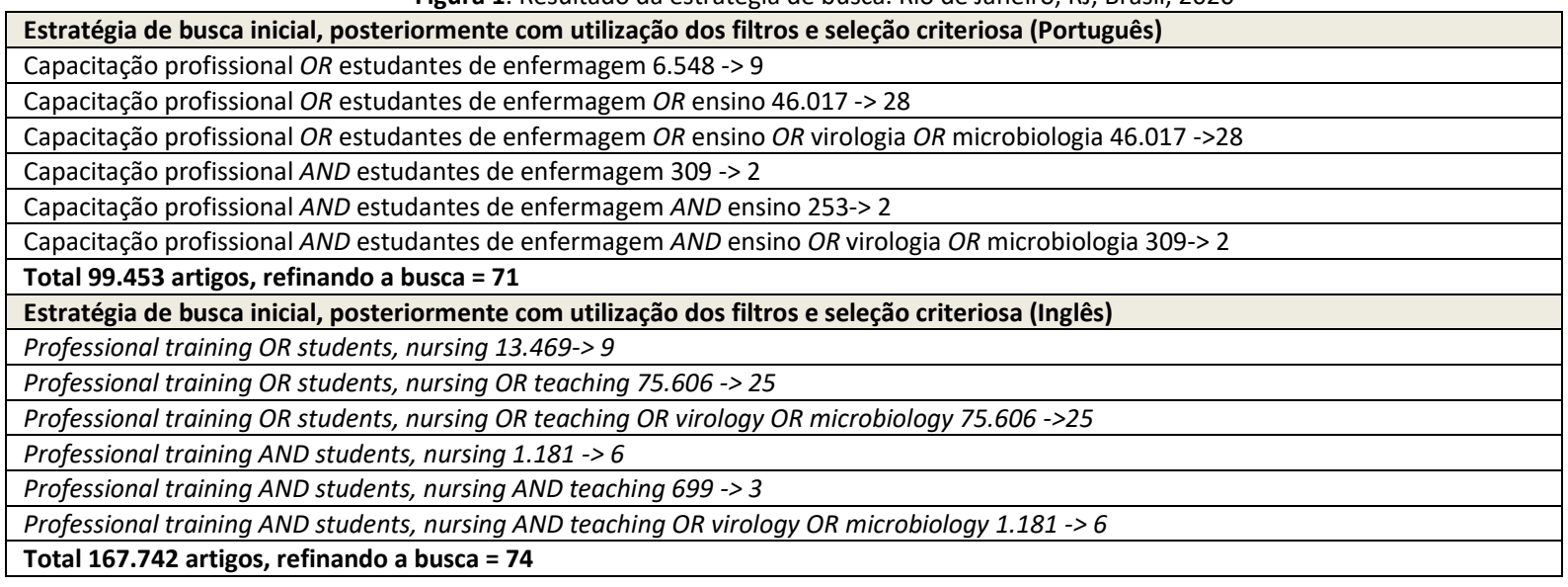

Posteriormente, foi elaborado um questionário com perguntas fechadas e abertas para que auxiliou no processo de seleção dos artigos. Essas perguntas tratavam sobre os temas formação e qualificação profissional; ensino do componente curricular Virologia; curso de graduação em Enfermagem; e Pandemia. Os dados do questionário foram elencados em arquivo de documento no software Microsoft Word.
A etapa de apreciação foi processada por meio de leitura analítica das produções científicas selecionadas, para verificação da adesão à temática proposta. Em seguida, realizou-se uma rigorosa interpretação dos resultados. Após isso, houve apresentação da revisão dos estudos, da qual foram eleitos nove artigos, que originou na junção do Total 1 e o Total 2 (Figura 2). Isso mostra como resultado final da busca atendeu integralmente ao rigor metodológico proposto.

Figura 2. Resultado final da busca. Rio de Janeiro, RJ, Brasil, 2020 Estratégia de busca inicial, posteriormente com utilização dos filtros e seleção criteriosa (Português) Capacitação profissional $O R$ estudantes de enfermagem $9->0$ Capacitação profissional $O R$ estudantes de enfermagem $O R$ ensino 28 -> 2

Capacitação profissional $O R$ estudantes de enfermagem $O R$ ensino $O R$ virologia $O R$ microbiologia $28->2$ Capacitação profissional $A N D$ estudantes de enfermagem $2 \rightarrow 0$

Capacitação profissional $A N D$ estudantes de enfermagem $A N D$ ensino $2 \rightarrow 0$

Capacitação profissional $A N D$ estudantes de enfermagem $A N D$ ensino $O R$ virologia $O R$ microbiologia 2 -> 0

Total 1 = 71 artigos, refinando a busca $=4$ e retirando duplicados $=2=$ Total 2

Estratégia de busca inicial, posteriormente com utilização dos filtros e seleção criteriosa (Inglês)

Professional training OR students, nursing $9 \rightarrow 5$ 
A formação profissional do enfermeiro: entrelace entre ensino da virologia e SARS-CoV-2 em uma universidade pública Chrizostimo MM, Caldas CP, Brito NR, Sánchez MCO, Souza DF, Xavier ML, Silva ARG, Silva JON, Ferraz VHG

\begin{tabular}{|l|}
\hline Professional training OR students, nursing OR teaching $25->6$ \\
\hline Professional training OR students, nursing OR teaching OR virology OR microbiology $25->6$ \\
\hline Professional training AND students, nursing $6->5$ \\
\hline Professional training AND students, nursing AND teaching $3->2$ \\
\hline Total $2=74$ artigos, refinando a busca $=14$ e retirando duplicados $=7=$ Total 7 \\
\hline
\end{tabular}

Nesse contexto, o tratamento dos dados foi realizado por meio da análise de conteúdo, com ênfase nas mensagens e nas comunicações as quais emergiram a categoria temática: a relevância do ensino da Virologia na formação do enfermeiro, ao considerar a construção da competência deste profissional para a atuação durante os tempos de Pandemia, que tem como propósito ter o paciente como protagonista do atendimento. A seguir, são identificadas as nove produções científicas elegidas para a realização de análise (Quadro 1).

Quadro 1. Produções científicas selecionadas por autores e base de dados. Rio de Janeiro, RJ, Brasil, 2020

\begin{tabular}{|c|c|c|c|}
\hline $\begin{array}{c}\text { Produção } \\
\text { Científica (PC) }\end{array}$ & Título do Artigo & Autores & $\begin{array}{c}\text { Base de } \\
\text { dados }\end{array}$ \\
\hline PC1 & $\begin{array}{l}\text { Interprofessional health education: the experience } \\
\text { of the Federal University of Sao Paulo, Baixada } \\
\text { Santista campus, Santos, Brazil }\end{array}$ & $\begin{array}{l}\text { Batista, Nildo Alves; Rossit, Rosana } \\
\text { Aparecida Salvador; Batista, Sylvia Helena } \\
\text { Souza da Silva; Silva, Carla Cilene Baptista } \\
\text { da; Uchôa-Figueiredo, Lúcia da } \\
\text { Rocha; Poletto, Patricia Rios. }\end{array}$ & LILACS \\
\hline PC2 & $\begin{array}{l}\text { Factors associated with student learning processes } \\
\text { in primary health care units: A questionnaire study }\end{array}$ & $\begin{array}{l}\text { Elisabeth Bos, Hassan Alinaghizadeh, Mikko } \\
\text { Saarikoski, Päivi Kaila }\end{array}$ & MEDLINE \\
\hline PC3 & $\begin{array}{l}\text { Percepções de profissionais sobre o aprendizado } \\
\text { de estudantes de graduação na atenção básica }\end{array}$ & $\begin{array}{l}\text { Codato, Lucimar Aparecida } \\
\text { Britto; Garanhani, Mara Lúcia; González, } \\
\text { Alberto Durán. }\end{array}$ & LILACS \\
\hline PC4 & $\begin{array}{l}\text { Formação acadêmica para o SUS x competência } \\
\text { pedagógica do formador: algumas considerações } \\
\text { para o debate }\end{array}$ & $\begin{array}{l}\text { Damiance, Patrícia Ribeiro Mattar; Panes, } \\
\text { Vanessa Bertassi Clivelaro; Caldana, Magali } \\
\text { de Lourdes; Bastos, José Roberto de } \\
\text { Magalhães. }\end{array}$ & LILACS \\
\hline PC5 & $\begin{array}{l}\text { PET-Health as inducer of professional education to } \\
\text { Unified Health System }\end{array}$ & $\begin{array}{l}\text { Farias-Santos, Bárbara Cássia de } \\
\text { Santana; Noro, Luiz Roberto Augusto. }\end{array}$ & MEDLINE \\
\hline PC6 & $\begin{array}{l}\text { Teaching-service-community integration in practice } \\
\text { scenarios of interdisciplinary Health Education: an } \\
\text { experience of the Work Education for Health } \\
\text { Program (PET-Health) in Southern Bahia }\end{array}$ & $\begin{array}{l}\text { Faria, Lina; Quaresma, Márcia Alves; Patiño, } \\
\text { Rafael Andrés; Siqueira, Raquel; Lamego, } \\
\text { Gabriela. }\end{array}$ & LILACS \\
\hline PC7 & $\begin{array}{l}\text { Nursing training and their approximation to the } \\
\text { assumptions of the National Curriculum Guidelines } \\
\text { and Primary Health Care }\end{array}$ & Magnago, Carinne; Pierantoni, Celia Regina. & LILACS \\
\hline PC8 & $\begin{array}{l}\text { Nursing training and professional practice: an } \\
\text { integrative review of literature }\end{array}$ & $\begin{array}{l}\text { Bianca Joana Mattia, Maria Elisabeth } \\
\text { Kleba, Marta Lenise do Prado }\end{array}$ & MEDLINE \\
\hline PC9 & $\begin{array}{l}\text { Health education: learning from professional } \\
\text { practice }\end{array}$ & $\begin{array}{l}\text { Daniela Martinez Fayer Nalom, Joyce } \\
\text { Fernanda Soares Albino Ghezzi, Elza de } \\
\text { Fátima Ribeiro Higa, Cássia Regina } \\
\text { Fernandes Biffe Peres, Maria José Sanches } \\
\text { Marin }\end{array}$ & MEDLINE \\
\hline
\end{tabular}

\section{Resultados e Discussão}

Para apresentação dos resultados e realização das discussões aborda-se, primeiramente, a formação profissional do enfermeiro que está pautada nas DCNs e nos atos normativos que são instituídos pela CNE, que regulamentam o ensino. Sendo assim, o curso de graduação de Enfermagem deve vislumbrar o ensino da Virologia com o olhar para a COVID-19, ao considerar que o ensino é direcionado tendo o SUS como foco e articulação entre os seus princípios norteadores ${ }^{2,8,9}$.

Deste modo, pretende-se estabelecer o processo de ensino-aprendizagem pelos docentes e que o egresso, ao longo de sua carreira, atue de maneira eficiente, eficaz, com o rigor científico e o compromisso social. Com isso, se estabelece, independente da área escolhida, que todos irão possuir os mesmos objetivos e terão o paciente como protagonista de todo atendimento ${ }^{2,8,9}$.

Por meio desse pensamento, observa-se que em abril de 2004 foi instituída a Lei no 10.861 que criou o Sistema
Nacional de Avaliação da Educação Superior (SINAES), que possui como ferramenta avaliativa o Exame Nacional de Desempenho dos Estudantes (ENADE). O objetivo desta lei foi oferecer parâmetros, que variam de um a cinco, para que fossem mensurados a qualidade do ensino ministrado, dos professores, alunos e instituições. Dessa maneira, é possível realizar rankings comparativos entre diferentes cursos e verificar também os conhecimentos adquiridos pelo graduando ao decorrer de sua formação ${ }^{10}$. Entretanto, ainda se observa a necessidade de entrelaçar o ensino da Virologia com as pandemias.

Já em setembro de 2008, foi sancionada a Lei n.으 11.788 com o intuito de regularizar os estágios, que podem variar quanto ao grau de escolaridade, mas possuem a base fundamental de preparar o aluno para o ciclo profissional. O Programa de Educação pelo Trabalho para Saúde (PETSaúde) é uma iniciativa do Ministério da Saúde, que visa a articular a atuação do profissional de saúde junto com estudantes de graduação. É demonstrada também uma 
A formação profissional do enfermeiro: entrelace entre ensino da virologia e SARS-CoV-2 em uma universidade pública Chrizostimo MM, Caldas CP, Brito NR, Sánchez MCO, Souza DF, Xavier ML, Silva ARG, Silva JON, Ferraz VHG

relação positiva entre estudantes que participaram do programa e realizaram o ENADE. Com isso, fica evidente a importância da parte prática junto com a teoria, principalmente quando se está frente a COVID-19, o que influencia a formação do estudante ${ }^{11-14}$.

O currículo do curso de graduação de Enfermagem da Escola de Enfermagem Aurora de Afonso Costa (EEAAC) da Universidade Federal Fluminense (UFF) possui a duração de cinco anos, o período é integral e o aluno, ao final de sua formação, será considerado bacharel em Enfermagem. A matriz referencial baseia-se na escola francesa, que tem a perspectiva construtivista associada à matriz críticoemancipatória ${ }^{15}$.

O curso é dividido em dois ciclos, o básico e o profissional. No que diz respeito ao ciclo básico, as disciplinas encontram-se inseridas nos diversos cursos da saúde e o ciclo profissional contêm a especificidade da profissão. Sendo primordial que exista relação entre ambas às partes, configurando-se no currículo a integração entre a teoria e a prática ${ }^{15}$.

É necessário também que a graduação não esteja atrelada apenas a questões individuais, mas com os aspectos socioculturais e históricos, uma vez que se faz necessário aproximação com as demandas exigidas pela sociedade como um todo. Para isso, é crucial que seja vislumbrada a articulação entre o ensino da Virologia com os fatos e agravos que a população vivencia com a Pandemia. Bem como, providenciar os meios adequados para o ensino do aluno, de modo a fazer com que ele seja o protagonista de seu processo de aprendizagem. É fundamental que a metodologia antiga onde o conhecimento é apenas transmitido pelo professor, unidirecional, seja descartada e que o ensino seja pautado no compartilhamento e construção entre os sujeitos ${ }^{15,16}$.

Ainda é crucial que o aluno possua competências, que variam de geral à específicas, adquiridas e construídas ao longo da formação, como afirma o sociólogo Philippe Perrenoud. Desse modo, o docente deve mostrar inúmeras possibilidades para o aluno, estabelecendo uma relação efetiva e auxiliá-lo sempre que necessário, uma vez que o professor possui o papel de educador em uma sala de aula e de referência para o estudante. Em detrimento disso, a motivação para estudo foi pautada nesse fato, além de estimular também a educação continuada, para que o futuro enfermeiro permaneça investindo na busca de conhecimento após a graduação, e, que tal prática esteja presente no cotidiano do profissional $\left.\right|^{6,17-19}$.

Assim, cabe registrar que nessa graduação, a oferta de conhecimento que ocorre na disciplina de Virologia é de suma importância para ser ministrada durante o quarto período do curso de enfermagem da UFF, com a carga horária de 50 horas, sendo 40 de teoria e 10 prática. As aulas são articuladas com as visitas técnicas supervisionadas a laboratórios e o acompanhamento de rotina por meio da realização de técnicas variadas, além da realização de seminários sobre diferentes conteúdos e atividades diversas. Desse modo, é possível que ocorra uma integração efetiva, fazendo com que o aluno seja impulsionado a entender conceitos abstratos, que não podem ser visualizados a olhos nus e que por meio da prática é possível entender de maneira mais clara e objetiva ${ }^{20}$.

Os objetivos da disciplina consistem em aprofundar os conhecimentos empíricos que foram adquiridos ao longo da vida e trajetória do estudante, tanto no âmbito individual quanto no coletivo, além dos conteúdos novos. Isso possui o caráter científico, a fim de garantir que o assunto ministrado em sala de aula esteja dentro dos padrões necessários e que o conhecimento a ser compartilhado amplie a visão do estudante. O intuito é fazer com que o aluno consiga assimilar conceitos que serão relevantes para exercer sua profissão, utilizando para isso diferentes métodos ${ }^{20}$.

Com isso, a ementa contempla visitas técnicas supervisionadas, acompanhamento de rotina laboratorial, realização de seminários e a compreensão conteúdo teórico, possibilitando que o aluno tenha contato com o conteúdo que não está restrito apenas a sala de aula, resultando em um processo de ensino-aprendizagem mais dinâmico. Tornase evidente que, desse modo, tanto o professor quanto o aluno são agentes da educação, além de estimular o trabalho em equipe entre os colegas de classe. $O$ conhecimento passa a ser construído ao longo da disciplina, com o propósito final de que o aluno carregue consigo alguns conceitos fundamentais que irão nortear sua trajetória ${ }^{20}$.

Existem inúmeros tipos de vírus e eles realizam diversas mutações ao longo do tempo, fato esse que aumenta sua variabilidade genética e a probabilidade de sucesso em sua disseminação. Desse modo, em sala de aula é necessário o estabelecimento de parâmetros para selecionar os principais tópicos que serão abordados pelo professor, para que o ensino seja dinâmico e não cansativo. É preciso tratar sobre as características gerais de cada tipo de vírus, diferentes classes, métodos de replicação viral, vacinas, como o sistema imunológico responde, mecanismos de controle de infecção, dentre outros assuntos ${ }^{4}$.

Nesse contexto, esses tempos de Pandemia onde há maciça frequência de veiculação de notícias falsas, é fundamental que o enfermeiro, como educador, se empodere desse papel e dissemine as informações corretas. A perspectiva é a de fazer com que a população receba o conhecimento de maneira simples, objetiva e verdadeira. Sendo importante também que seja disseminado o aprendizado sobre medidas de proteção e conceitos abstratos sobre o que seria um vírus, quarentena, medidas restritivas etc. Desse modo, se tem uma sociedade que de fato compreende a gravidade da situação e que possui o conhecimento adequado, tornando-se mais fácil e assertiva a atuação na prevenção e erradicação de uma doença que é causada por vírus.

Assim, percebe-se que o ensino da Virologia possui relevância para a formação do enfermeiro, ao considerar a construção da competência deste profissional para a atuação durante o período de Pandemia, que precisa ter o paciente como protagonista do atendimento.

O sociólogo Philippe Perrenoud afirma que as competências e habilidades são construídas ao longo da formação. Desse modo, ao estudar Virologia, o futuro enfermeiro poderá atuar de modo efetivo na sua profissão, com a associação dos diferentes conteúdos que foram 
A formação profissional do enfermeiro: entrelace entre ensino da virologia e SARS-CoV-2 em uma universidade pública Chrizostimo MM, Caldas CP, Brito NR, Sánchez MCO, Souza DF, Xavier ML, Silva ARG, Silva JON, Ferraz VHG

ministrados e que estão entrelaçados entre si. Deve-se entender também a necessidade de estar o tempo todo vigilante, realizando as medidas de precaução necessárias, as funcionalidades e importância dos Equipamentos de Proteção Individual (EPI), higienização das mãos, esterilização, dentre outros aspectos. Além de trazer inúmeros benefícios não apenas para si, mas para a sociedade como um todo ${ }^{6}$.

Cabe aqui registrar que, em tempos de pandemia da COVID-19, o profissional de saúde atua na linha de frente e fica constantemente exposto ao vírus, particularmente, os profissionais de Enfermagem, que passam mais tempo com cada paciente. Desse modo, com o aprendizado e instrução adquiridos durante a graduação, o profissional terá a expertise necessária para tomar as devidas precauções, tendo o devido cuidado consigo e com o outro. Ademais, ele poderá instruir familiares, amigos e pacientes sobre quais medidas são corretas, o que deve ser evitado e ajudar no que for necessário.

Esse fato tem mostrado para a sociedade a real importância desses profissionais, uma vez que muitos setores ainda consigam exercer suas atividades em casa, os enfermeiros precisam sair todos os dias para cuidar dos pacientes e, ao mesmo tempo, se colocar em risco. Isto é evidente ao se verificar a quantidade de profissionais da saúde que vieram a óbito e foram contaminados com a doença.

A Pandemia mudou a vida de todos, com variação apenas no grau de impacto e seus efeitos, que poderão ser prolongados. Atualmente, no Brasil, mais de meio milhão de pessoas morreram em decorrência da COVID-19. Segundo dados disponibilizados pelo Ministério da Saúde, a pandemia ceifou a vida de 500.800 brasileiros, sendo contabilizadas 2.301 mortes e 82.288 novos casos confirmados em 24 horas, além de 1.199.101 ocorrências que estão sendo monitoradas ${ }^{21}$.

Esse indicador pode ser relacionado a uma série de fatores, como o elevado índice de contaminação do vírus e a não realização das medidas necessárias por parte das autoridades sanitárias e da própria população. Lamentavelmente, o país passa por duas crises que colabora para o aumento do número de novos casos: a do vírus e a da ignorância. Constata-se que "essa perigosa combinação expõe mais pessoas ao risco de contágio e dificulta ainda mais as estratégias de prevenção da doença" e, por isso, ocorre a "(...) alta ocupação de leitos de UTI e a escassez de medicamentos para intubação, o que aumenta ainda mais a pressão sobre os trabalhadores de saúde"21:2.
Além disso, o país enfrenta grandes dificuldades para conter o vírus e impedir sua propagação, que ainda não está controlada por meio de vacinação. É preciso destacar que, até "o momento, foram enviadas a estados e municípios 115,135 milhões de doses de vacinas. Desse total, foram aplicadas 85.390 milhões de doses, sendo 61.270 milhões da primeira dose e 24.120 da segunda dose"21:2.

É evidente, portanto, que a pandemia SARS-COV-2 trouxe um grande desafio para a formação profissional na área da saúde. Trazendo à tona a relevância da Virologia para o cotidiano da vida profissional, visto que o ano de 2020 permanecerá na história da educação desses profissionais como o ano em que foi possível estabelecer um claro entrelaçamento entre a Virologia, que é ensinada na academia e a da realidade.

\section{Considerações Finais}

De acordo com os objetivos estabelecidos para o estudo, foi possível compreender o entrelaces entre o ensino teórico da Virologia e a realidade da vida profissional. Evidenciando-se a ocorrência de maior contato com o cotidiano de sua futura profissão faz com que o aluno consiga melhor compreender a disciplina e criar conexões mais efetivas entre teoria e prática. $O$ docente possui papel crucial na vida acadêmica do estudante, de modo a construir o conhecimento em sincronia com a realidade, pautado no aluno como sujeito principal do processo ensinoaprendizagem.

Também foi esclarecida a importância da disciplina de Virologia na graduação do futuro enfermeiro, pois diversos conceitos e práticas associadas ao cuidado serão necessários em sua atuação profissional. De certo, as competências e habilidades serão desenvolvidas ao longo do tempo de experiência, possibilitando que o enfermeiro execute de maneira plena o seu papel, para atender a sociedade com excelência e continuar empreendendo esforço em outros cursos, tendo em vista que o conhecimento é dinâmico.

Ademais, em tempos de Pandemia, os impactos são mensurados em escala global e existe a necessidade de avanços, cuidado e vigilância constante. Sendo fundamental que os profissionais de saúde trabalhem em equipe, visando sempre o melhor para o grupo de trabalho e para os pacientes e familiares. Para isso, devem ser utilizados todos os recursos necessários e a promoção do empoderamento da população com informações efetivas e educativas.

\section{Referências}

1. Chrizostimo MM, Brandão AAP. La formación profesional del enfermero: 'estado del arte'. Enfermería Global. 2015;14(4):414-445. https: //doi.org/10.6018/eglobal.14.4.208841

2. Ministério da Educação (BR). Conselho Nacional de Educação. Resolução CNE/CES n. 3, de 7 de novembro de 2001. Institui Diretrizes Curriculares Nacionais do Curso de Graduação em Enfermagem [Internet]. Brasília (DF): Ministério da Educação; 2001 [acesso em 13 out 2020]. Disponível em: http://portal.mec.gov.br/cne/arquivos/pdf/CES03.pdf 
A formação profissional do enfermeiro: entrelace entre ensino da virologia e SARS-CoV-2 em uma universidade pública Chrizostimo MM, Caldas CP, Brito NR, Sánchez MCO, Souza DF, Xavier ML, Silva ARG, Silva JON, Ferraz VHG

3. Silva RR, Lipari CC, Araujo MS, Silva LA, Silva MVG, Franco AS, Marta CB, Larrubia EO, Francisco MTR, Oliveira ES. Contribuições da monitoria em Fundamentos de Enfermagem II na formação acadêmica de estudantes de enfermagem: relato de experiência. Glob Acad Nurs. 2021;2(1):e79. https://doi.org/10.5935/2675-5602.20200079

4. Santos NSDO, Romanos MTV, Wigg MD. Virologia humana. 3.ed. Rio de Janeiro: Guanabara Koogan; 2015.

5. Yuen KS, et. al. SARS-CoV-2 and COVID-19: The most important research questions. Cell \& Bioscience. 2020;10(1):1-5. DOI: 10.1186/s13578-020-00404-4

6. Perrenoud P, Thurler MG. As competências para ensinar no século XXI: a formação dos professores e o desafio da avaliação. Porto Alegre: Artmed; 2009.

7. Mendes KDS, Silveira RCDCP, Galvão CM. Revisão integrativa: método de pesquisa para a incorporação de evidências na saúde e na enfermagem. Texto Contexto Enferm. 2008;17(4):758-764. https://doi.org/10.1590/S0104-07072008000400018

8. Batista NA, et. al. Interprofessional health education: the experience of the Federal University of Sao Paulo, Baixada Santista campus, Santos, Brazil. Interface (Botucatu). 2018;22:1705-1715. https://doi.org/10.1590/1807-57622017.0693

9. Damiance PRM, et. al. Formação acadêmica para o SUS x competência pedagógica do formador: algumas considerações para o debate. Salusvita [Internet]. 2016 [acesso em 13 out 2020];35(3):453-74. Disponível em: https://secure.unisagrado.edu.br/static/biblioteca/salusvita/salusvita_v35_n3_2016_art_11.pdf

10. Presidência da República (BR). Casa Civil. Lei n.o 10.861, de 14 de abril de 2004. Institui o Sistema Nacional de Avaliação da Educação Superior (SINAES) e dá outras providências [Internet]. Brasília (DF): Casa Civil. 2004 [acesso em 13 out 2020]. Disponível em: http://www.planalto.gov.br/ccivil_03/_ato2004-2006/2004/lei/I10.861.htm

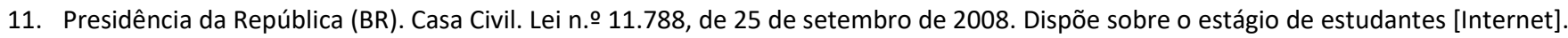
Brasília (DF): Casa Civil; 2008 [acesso em 13 out 2020] Disponível em: http://www.planalto.gov.br/ccivil_03/_ato20072010/2008/lei/l11788.htm

12. Farias-Santos BCDS, Noro LRA. PET-Health as inducer of professional education to Unified Health System. Ciência \& Saúde Coletiva. 2017;22:997-1004. https://doi.org/10.1590/1413-81232017223.15822016

13. Faria L, et. al. Teaching-service-community integration in practice scenarios of interdisciplinary Health Education: an experience of the Work Education for Health Program (PET-Health) in Southern Bahia. Interface. 2018;22(67):1257-1266. https://doi.org/10.1590/180757622017.0226

14. Mattia BJ, Kleba ME, Prado MLD. Nursing training and professional practice: an integrative review of literature. Rev Bras Enferm. 2018;71(4):2039-2049. https://doi.org/10.1590/0034-7167-2016-0504

15. Universidade Federal Fluminense (UFF). Projeto Pedagógico do Curso (PPC). Rio de Janeiro: Coordenação do Curso de Graduação em Enfermagem e Licenciatura / PROAC, 2004.

16. Nalom DMF, et. al. Health education: learning from professional practice. Ciência \& Saúde Coletiva. 2019;24:1699-1708. https://doi.org/10.1590/1413-81232018245.04412019

17. Codato LAB, Garanhani ML, González AD. Percepções de profissionais sobre o aprendizado de estudantes de graduação na Atenção Básica. Physis. 2017;27(3):605-619. https://doi.org/10.1590/S0103-73312017000300012

18. Magnago C, Pierantoni CR. Nursing training and their approximation to the assumptions of the National Curriculum Guidelines and Primary Health Care. Ciência \& Saúde Coletiva. 2020;25:15-24. https://doi.org/10.1590/1413-81232020251.28372019

19. Bos E, et. al. Factors associated with student learning processes in primary health care units: A questionnaire study. Nurse education today, 2015;35(1):170-175. DOI: 10.1016/j.nedt.2014.09.012

20. Universidade Federal Fluminense (UFF). Conteúdo dos estudos Virologia para Enfermagem. Rio de Janeiro: Pró Reitoria de Assuntos Acadêmicos, 2020.

21. Peduzzi P. Brasil registra mais de 500 mil mortos por COVID-19. Agência Brasil [Internet]. 2021 [acesso em 22 jun 2021 ]. Disponível em: https://agenciabrasil.ebc.com.br/ saude/noticia/2021-06/brasil-registra-mais-de-500-mil-mortos-por-covid-19 